\title{
MicroRNA-381/Hes1 is a potential therapeutic target for spinal cord injury
}

\author{
WENDONG RUAN*, GUANGZHI NING ${ }^{*}$, SHIQING FENG, SHIJIE GAO and YAN HAO \\ Department of Orthopedics, The General Hospital of Tianjin Medical University, Heping, Tianjin 300052, P.R. China
}

Received January 11, 2017; Accepted January 17, 2018

DOI: $10.3892 /$ ijmm.2018.3658

\begin{abstract}
The aim of the present study was to investigate whether microRNA-381 is a potential therapeutic target for spinal cord injury (SCI) and its possible mechanism. Reverse transcription quantitative polymerase chain reaction (qPCR) for mRNA expression was used to analyze the changes of microRNA-381 expression. Cell viability and cell apoptosis were measured using 3-(4,5-dimethylthiazol-2-yl)-2,5-diphenyltetrazolium bromide (MTT) assay and flow cytometry. Caspase-3 activity was measured using caspase-3 activity kit, and western blot analysis was used to measure the protein expression of neurogenic locus notch homolog protein 1 (Notch1), notch 1 intracellular domain (NICD) and transcription factor HES-1 (Hes1). The data showed that microRNA-381 expression of model SCI rats was downregulated compared with that of control rats. Overexpression of microRNA-381 promoted cell proliferation, and inhibited apoptosis and caspase-3 and apoptosis regulator BAX (Bax) protein expression in neurocytes. Overexpression of microRNA-381 also increased Wnt and $\beta$-catenin protein expression, and suppressed the protein expression of Notch1, NICD and Hes1 in neurocytes. Wnt inhibitor, Wnt-C59 (1 $\mu \mathrm{mol} / \mathrm{l})$, inhibited cell proliferation, promoted apoptosis and caspase- 3 and Bax protein expression, suppressed $\beta$-catenin protein expression and induced Hes1 protein expression in neurocytes following microRNA-381 overexpression. Notch inhibitor, FLI-06 (1 $\mu \mathrm{mol} / \mathrm{l})$, promoted cell proliferation, inhibited apoptosis and caspase- 3 and Bax protein expression, and suppressed NICD and Hes1 protein expression in neurocytes following microRNA-381 overexpression. Thus, this study showed that overexpression of microRNA-381 promotes cell proliferation of neurocytes in SCI via Hes1 expression, which may be a novel important mechanism for SCI in clinical applications.
\end{abstract}

Correspondence to: Mr. Wendong Ruan, Department of Orthopedics, The General Hospital of Tianjin Medical University, 154 Anshan Road, Heping, Tianjin 300052, P.R. China

E-mail: ruanwendong154@163.com

${ }^{*}$ Contributed equally

Key words: microRNA-381, transcription factor HES-1, spinal cord injury, neurogenic locus notch homolog protein 1 , Wnt

\section{Introduction}

As a common motor system trauma in the clinic, $\mathrm{SCI}$ has been observed to induce various degrees of extremity swelling or paraplegia and loss of ability to work, bringing a tremendous burden on society and the patient's family (1). The epidemiological data in American regions demonstrate that traffic accidents and high-altitude falls are the major causes of injury, with cases more often occurring in young men, with an average age of 30 years old. The average annual morbidity is 420 million cases, and the number of patients is increasing at a speed of 10,000 individuals each year (2). In the field of sports injury, domestic and overseas scholars have made unremitting efforts and actively investigated the novel therapeutic strategies for SCI, in spite of the tremendous difficulties in post-SCI repair $(3,4)$.

MicroRNA (miRNA) is a class of short non-coding single-stranded micromolecule that is distributed among multiple animals and plants, and is highly conserved in evolution (5). It has been indicated in bioinformatics predictions that miRNA at least regulates the expression of human protein-coding genes. The discovery of miRNA has provided a novel thought process for illustrating the physiopathological mechanisms and treatments of human diseases (2). miRNA is widely distributed in the central nervous system (6). miRNA has been suggested to be associated with the development of the neural system, as well as the differentiation and proliferation of nerve cells (7) As is also found in numerous neurodegenerative diseases, miRNA is involved in their pathophysiological processes and the expression of miRNAs cause changes in SCI (8). Abnormal expression of miRNA can be observed in central nervous system injury, and may take part in the pathological processes of the central nervous system (9).

The secondary injury mechanisms of acute SCI include oxygen free radicals, excitatory amino acids, vascular injury, inflammation and cell apoptosis. Of these, cell apoptosis serves an important role in SCI, but the mechanism remains to be further investigated (10).

The Hes family basic helix-loop-helix (bHLH) transcription factor 1 (Hes1) gene exists in the majority of mammals, expressed to a large extent in the epithelial and neuroepithelial cells during the embryonic development process (11). As a bHLH DNA-binding protein, neurogenic locus notch homolog protein 1 (Hesl protein) can block the activation pathway through binding with target DNA or other HLH proteins (including achaete-scute homolog 1 and E47) directly, thus inhibiting 
transcription and finally inhibiting cell differentiation (12). During the development of the ervous system, Hes1 participates in regulating the differentiation and proliferation of neural stem cells (13). The role of the Hesl gene in neural stem cells has mainly been identified through gain- and loss-of-function research (14). Hes1 gene knockout fetal rats develop severe neurulation defects, and die during the embryonic period or at birth (15). Knockout of the Hes1 gene during development of the nervous system in the chicken embryo will lead to premature differentiation and maturation of neural stem cells, resulting in microcephaly or anencephaly (15). Appropriate Hes1 expression can accurately regulate the genesis of the neural system and cellularity, which facilitates the formation of nerve cells and functional zones with various functions (16). Increased Hes1 expression following neuronal formation can accelerate the differentiation of astrocytes, which is in contrast to its role in inhibiting the differentiation of neural stem cells in the early stage, demonstrating that Hes1 serves various roles in regulating the differentiation of neural stem cells during various periods (16). As previously shown, the endogenous neural stem cells can differentiate into mature nerve cells under the regulation of signaling molecules (16). The present study examined whether microRNA-381 is a potential therapeutic target for SCI and investigated its possible mechanism.

\section{Materials and methods}

Ethical approval and animal care. Male Sprague-Dawley rats (6 weeks old; 220-250 g) were purchased from the Animal Experimental Center of Tianjin Medical University and maintained at $24^{\circ} \mathrm{C}$, in $55 \%$ humidity on a 12 -h light/dark cycle, with standard rodent chow and access to water ad libitum. All rats were acclimatized for at least 1 week prior to the experiments. The Regional Committee for Medical and Health Research Ethics (Health Authority Southern and Eastern Norway) and the Regional Ethics Committee at Tianjin Medical University (Tianjin, China) approved the study protocol. All rats $(n=12)$ were randomly assigned into 2 groups: control and SCI model group ( $\mathrm{n}=6$ rats/group).

Rats were intraperitoneally anesthetized with $10 \%$ chloral hydrate $(300 \mathrm{mg} / \mathrm{kg})$, and then maintained at $37.0 \pm 0.5^{\circ} \mathrm{C}$ with an infrared heat lamp and a heating pad. To induce the SCI model, a 2F-Fogarty balloon catheter was inserted via the left femoral artery into the proximal descending thoracic aorta. The site of insertion is was $\sim 11 \mathrm{~cm}$ to the proximal descending thoracic aorta. Next, $0.05 \mathrm{ml}$ distilled water was injected into injury points to induce SCI. The arterial catheters were removed and the incisions were sutured after $24 \mathrm{~h}$.

Reverse transcription-quantitative polymerase chain reaction $(q P C R)$ for $m R N A$ expression. RNA was extracted from tissues samples and PC12 cells using TRIzol reagent (Invitrogen; Thermo Fisher Scientific, Inc., Waltham, MA, USA). RNA (200 ng) was prepared and compound First Strand cDNA was obtained using SuperScript II reverse transcriptase (Takara Biotechnology Co., Ltd., Dalian, China). $1 \mu$ l PrimeScript RTase, $4 \mu 1$ 5X PrimeScript buffer, $0.5 \mu 1$ RNase inhibitor, $1 \mu \mathrm{l}$ dNTP mixture, $1 \mu \mathrm{l}$ Oligo(dT) Primer, $2 \mu 1$ random 6-mers, $19.5 \mu \mathrm{l}$ RNase-free $\mathrm{H}_{2} \mathrm{O}$ were used. Relative mRNA expression was determined by qPCR (StepOnePlus RT-PCR
System; Applied Biosystems, Foster City, CA, USA) using Power SYBR-Green PCR master mix (Takara Biotechnology Co., Ltd.), with the following cycling parameters: $10 \mathrm{~min}$ at $95^{\circ} \mathrm{C}$, followed by 40 cycles of $15 \mathrm{sec}$ at $95^{\circ} \mathrm{C}$ and $30 \mathrm{sec}$ at $60^{\circ} \mathrm{C}$. The quantification cycle $(\mathrm{Cq})$ was determined and calculations were computed using the $2^{-\Delta \Delta \mathrm{Cq}}(17)$. Primer pairs used in this study were: U6 forward, 5'-CTCGCTTCGGCAGCA CA-3' and reverse, 5'-AACGCTTCACGAATTTG CGT-3'; and microRNA-381 forward, 5'-AGTCTATACAAGGGCAAGC T CTC-3' and reverse, 5'-ATCCATGACAGATCCCTACCG-3'.

Cell line and cell culture. PC12 cells were provided by the Cell bank of the Chinese Academy of Sciences (Shanghai, China) and incubated in Dulbecco's modified Eagle's medium, supplemented with $10 \%$ fetal bovine serum (both from Gibco; Thermo Fisher Scientific, Inc.) and $1 \%$ penicillin/streptomycin (Sigma-Aldrich; Merck KGaA) at $37^{\circ} \mathrm{C}$ with $5 \% \mathrm{CO}_{2}$.

Transfection. Negative (5'-CCCCCCCCCCCCC-3' and 5'-CCCCCCCCCCCC-3') and microRNA-381 mimics (5'-TACTTAAAGCGAGGTT-3' and 5'-TCGAGAGACT CAT-3') were purchased from Sangon Biotech (Shanghai) Co., Ltd. (Shanghai, China). The PC12 cells were seeded into a 6 -well plate at a density of $1 \times 10^{6} /$ well and were transfected using $500 \mathrm{ng}$ mimics with Lipofectamine 2000 (Thermo Fisher Scientific, Inc.) at $37^{\circ} \mathrm{C}$ with $5 \% \mathrm{CO}_{2}$. After $4 \mathrm{~h}$ of transfection, the old medium was removed, and the cells were treated with Wnt-C59 (2 $\mu \mathrm{M}$; MedChemExpress, Monmouth Junction, NJ, USA) and FLI-06 (1 $\mu \mathrm{M}$; MedChemExpress) for $48 \mathrm{~h}$.

Cell viability. Subsequent to transfection for $48 \mathrm{~h}$, the PC12 cells were collected at $1,000 \mathrm{x}$ g for $10 \mathrm{~min}$ and seeded into a 96-well plate at a density of $1-2 \times 10^{3} /$ well, treated with $5 \mathrm{mg} / \mathrm{ml}$ 3-(4,5-dimethylthiazol-2-yl)-2,5-diphenyltetrazolium bromide (MTT) assay for $4 \mathrm{~h}$, and then the media were replaced with dimethyl sulfoxide $(150 \mu \mathrm{g})$. Optical density was analyzed using an mQuant Microplate Spectrophotometer (BioTek, Potton, UK) at a wavelength of $490 \mathrm{~nm}$.

Cell apoptosis assays using flow cytometry. Subsequent to transfection for 48 , the PC12 cells were seeded into a 6-well plate at a density of $1 \times 10 \%$ well, and stained with a $10-\mu 1$ Annexin $\mathrm{V}$ and $5-\mu \mathrm{l}$ propidium iodide staining kit (Invitrogen; Thermo Fisher Scientific, Inc.) for $15 \mathrm{~min}$ in the dark. Cell apoptosis was performed using flow cytometry (FACSVerse; BD Biosciences, Franklin Lakes, NJ, USA).

Caspase-3 activity. Subsequent to transfection for $48 \mathrm{~h}$, the PC12 cells were seeded into a 6-well plate at a density of $1 \times 10^{6} \%$ well, and incubated with Ac-DEVD-pNA for $1 \mathrm{~h}$ at $37^{\circ} \mathrm{C}$. Optical density was analyzed using an mQuant Microplate Spectrophotometer (BioTek) at a wavelength of $405 \mathrm{~nm}$.

Western blot analysis. Subsequent to transfection for $48 \mathrm{~h}$, the PC12 cells were seeded intoa 6-well plate at a density of 1x10\% $/$ well, extracted using lysis buffer and clarified by centrifugation for $20 \mathrm{~min}$ in a microcentrifuge at $4^{\circ} \mathrm{C}$. The total protein concentration was determined with the BCA protein assay kit (Beyotime Institute of Biotechnology, Shanghai, China) and the resulting supernatant (50 $\mu \mathrm{g}$ protein) was subjected to $8-10 \%$ sodium 


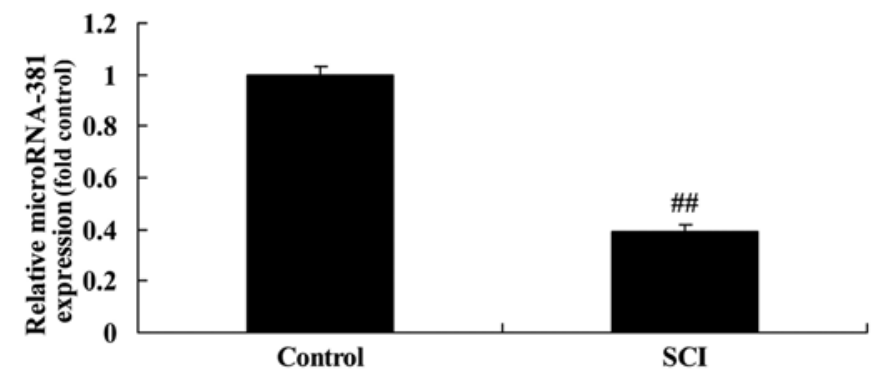

Figure 1. MicroRNA-381 expression of model SCI rats. MicroRNA-381 expression of model SCI rats, as determined by reverse transcription-quantitative polymerase chain reaction. ${ }^{\# \#} \mathrm{P}<0.01$ vs. control group. Control, control group; SCI, spinal cord injury model rat group.

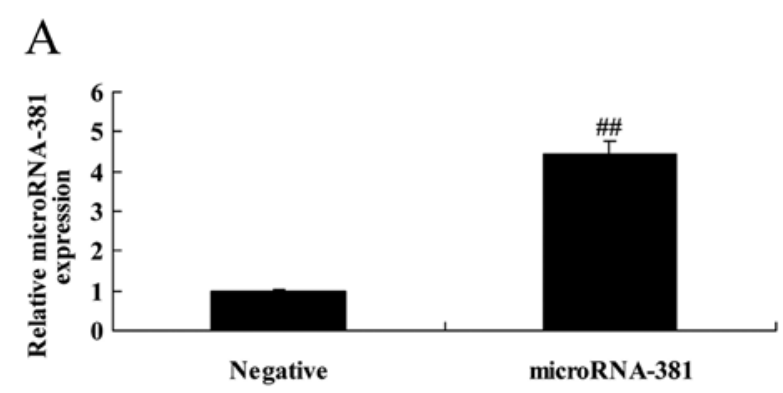

$\mathrm{B}$

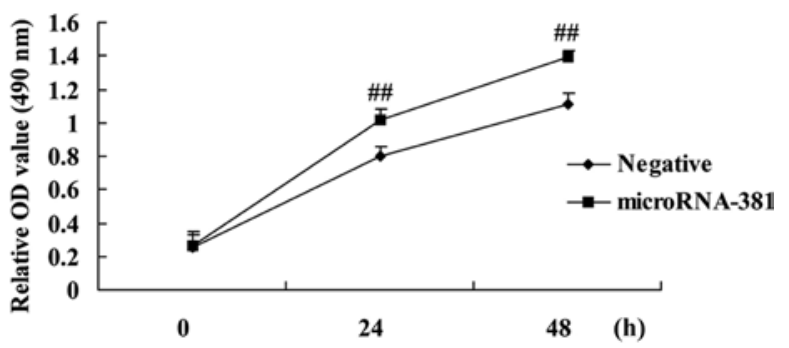

Figure 2. Overexpression of microRNA-381 promotes cell proliferation of neurocytes. (A) Overexpression of microRNA-381 and (B) promotion of cell proliferation of neurocytes. ${ }^{\# \#} \mathrm{P}<0.01$ vs. negative control group. Negative, negative control group; microRNA-381, microRNA-381 overexpression group; OD, optical density.

dodecyl sulfate-polyacrylamide gel electrophoresis. The separated proteins were transferred to polyvinylidene difluoride membranes (Amersham, Buckinghamshire, UK). The membranes were blocked with $5 \%$ skimmed milk for $1 \mathrm{~h}$ at $37^{\circ} \mathrm{C}$ and incubated with primary antibody against apoptosis regulator BAX (Bax; catalog no. sc-6236, 1:500 dilution), Wnt (catalog no. sc-292332; 1:500 dilution), $\beta$-catenin (catalog no. sc-7199, 1:500 dilution), neurogenic locus notch homolog protein 1 (Notch1; catalog no. sc-9170, 1:500 dilution) (all from Santa Cruz Biotechnology, Inc., Dallas, TX, USA), notch 1 intracellular domain (NICD; catalog no. ab83232, 1:500 dilution; Abcam, Cambridge, UK), Hes1 (catalog no. sc-25392, 1:500 dilution) and glyceraldehyde 3-phosphate dehydrogenase (catalog no. sc-25778, 1:1,000 dilution) (both from Santa Cruz Biotechnology, Inc.) at $4^{\circ} \mathrm{C}$ overnight. The proteins were incubated with anti-rabbit horseradish peroxidase-conjugated secondary antibody (sc-2004; Santa Cruz Biotechnology, Inc.), visualized using an enhanced chemiluminescence system (Pierce Co., Rockford, IL, USA) and analyzed using Image-ProPlus 6.0 software (Media Cybernetics, Inc., Rockville, MD,USA).
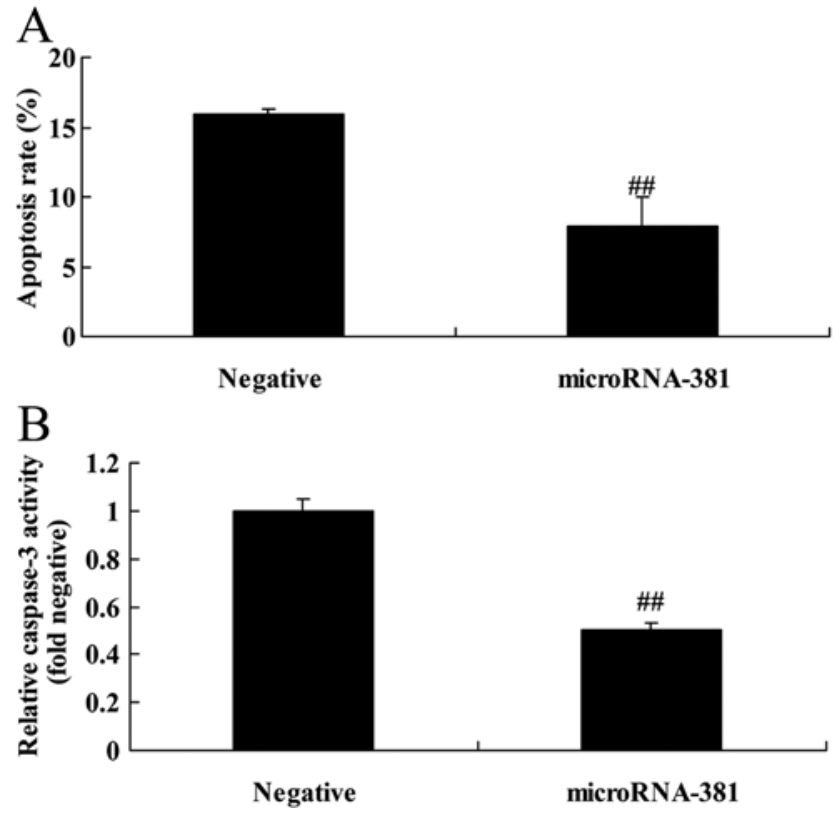

Figure 3. Overexpression of microRNA-381 inhibits apoptosis and caspase-3 activity of neurocytes. Overexpression of microRNA-381 inhibited (A) apoptosis and (B) caspase-3 activity of neurocytes. ${ }^{\# \#} \mathrm{P}<0.01$ vs. negative control group. Negative, negative control group; microRNA-381, microRNA-381 overexpression group.

Statistical analysis. All data are presented as the mean \pm standard deviation. Differences between groups were tested by the Student's t-test or one-way analysis of variance using the SPSS 17.0 program (SPSS, Inc., Chicago, IL, USA). P $<0.05$ was considered to indicate a statistically significant difference.

\section{Results}

MicroRNA-381 expression of model SCI rat. Firstly, the microRNA-381 expression was analyzed in the model SCI rat and the control group. Fig. 1 shows that the microRNA-381 expression of the model SCI rat group was downregulated compared with that of the control rat group $(\mathrm{P}<0.05)$.

Overexpression of microRNA-381 promotes cell proliferation of neurocytes. Next, microRNA-381 mimics were used to increase microRNA-381 expression in PC12 cells, and then an MTT assay was used to analyze the cell proliferation of neurocytes. As shown in Fig. 2, overexpression of microRNA-381 significantly promoted cell proliferation of PC12 cells in a time-dependent manner $(\mathrm{P}<0.05)$.

Overexpression of microRNA-381 inhibits apoptosis and caspase-3 activity of neurocytes. The study investigated the effects of microRNA-381 on the apoptosis mechanism of neurocytes. As shown in Fig. 3, overexpression of microRNA-381 significantly inhibited the apoptosis rate and caspase- 3 activity of the PC12 cells (both $\mathrm{P}<0.05$ ).

Overexpression of microRNA-381 inhibits Bax protein expression of neurocytes. To understand the molecular mechanism of action of microRNA-381 on Bax protein expression of neurocytes, Bax protein expression was measured using western blot analysis. Fig. 4 shows that the Bax protein expression of PC12 
A

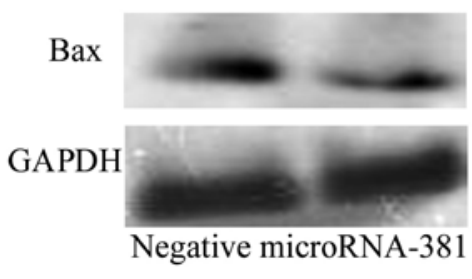

B

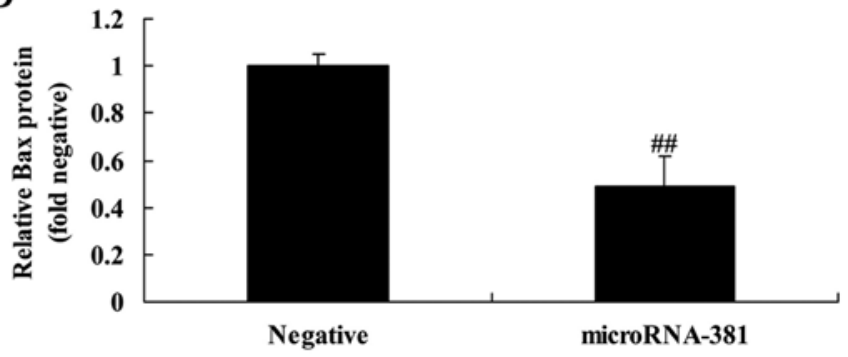

Figure 4. Overexpression of microRNA-381 inhibits Bax protein expression of neurocytes. Overexpression of microRNA-381 was shown to inhibit Bax protein expression, as determined using (A) western blot analysis and (B) statistical analysis of Bax protein expression of neurocytes. ${ }^{\#} \mathrm{P}<0.01$ vs. negative control group. Negative, negative control group; microRNA-381, microRNA-381 overexpression group; GAPDH, glyceraldehyde 3-phosphate; Bax, apoptosis regulator BAX.
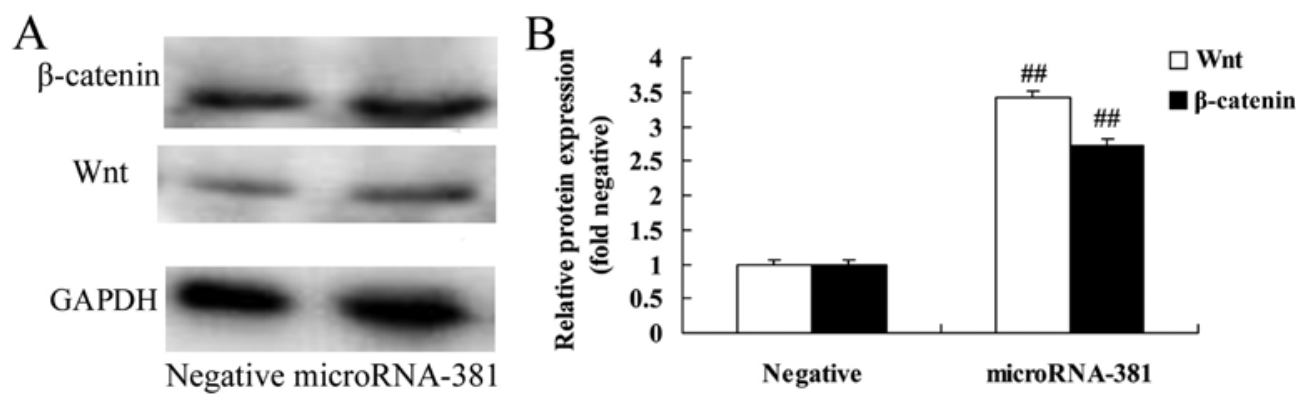

Figure 5. Overexpression of microRNA-381 increases Wnt and $\beta$-catenin protein expression of neurocytes. Overexpression of microRNA-381 was shown to inhibit Wnt and $\beta$-catenin protein expression, as determined using (A) western blot analysis and (B) statistical analysis of Wnt and $\beta$-catenin protein expression of neurocytes. ${ }^{\# \#} \mathrm{P}<0.01$ vs. negative control group. Negative, negative control group; microRNA-381, microRNA-381 overexpression group; GAPDH, glyceraldehyde 3-phosphate

A

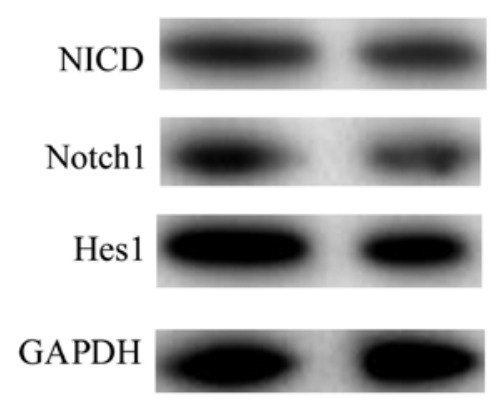

Negative microRNA-381
$\mathrm{B}$

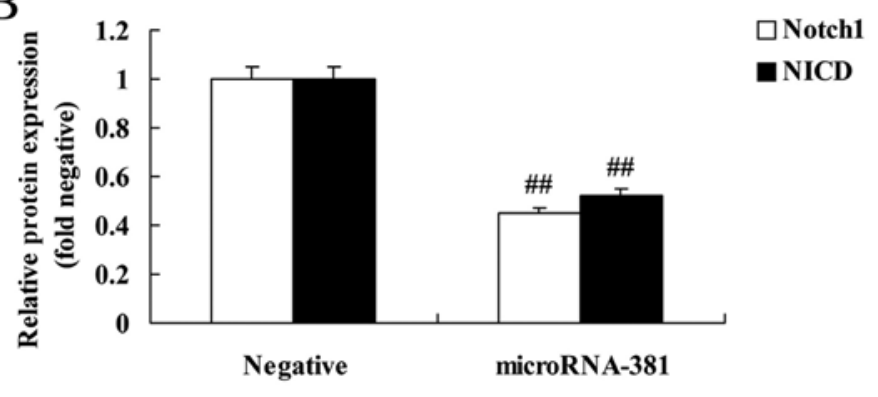

C

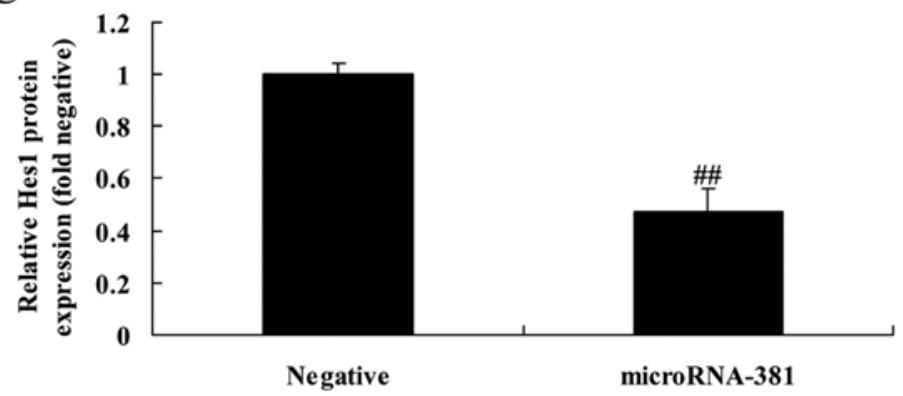

Figure 6. Overexpression of microRNA-381 suppresses the protein expression of Notch1, NICD and Hes1 of neurocytes. Overexpression of microRNA-381 was shown to inhibit Notch1, NICD and Hes1 protein expression, as determined using (A) western blot analysis and statistical analysis of (B) Notch1 and NICD, and (C) Hes1 protein expression of neurocytes. ${ }^{\# /} \mathrm{P}<0.01$ vs. negative control group. Negative, negative control group; microRNA-381, microRNA-381 overexpression group; GAPDH, glyceraldehyde 3-phosphate; Notch1, neurogenic locus notch homolog protein 1; NICD, notch 1 intracellular domain; Hes1, transcription factor HES-1.

cells was significantly suppressed by microRNA-381 overexpression $(\mathrm{P}<0.05)$.

Overexpression of microRNA-381 increases Wnt and $\beta$-catenin protein expression of neurocytes. To assess the function of microRNA-381 in the Wnt/ $\beta$-catenin signaling of neurocytes, western blot analysis was used to analyze Wnt and $\beta$-catenin protein expression. Fig. 5 indicates that overexpression of microRNA-381 significantly increased the Wnt and $\beta$-catenin protein expression of $\mathrm{PC} 12$ cells $(\mathrm{P}<0.05)$. 
A

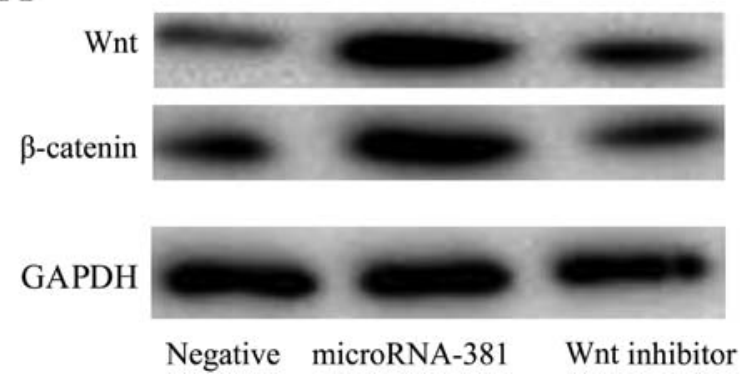

B

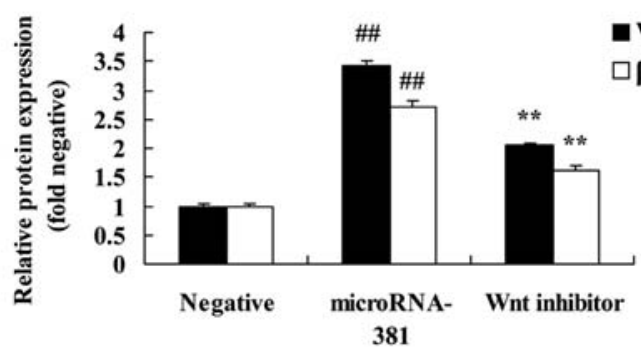

Figure 7. Inhibition of Wnt inhibits Wnt and $\beta$-catenin protein expression of neurocytes following microRNA-381 overexpression. Inhibition of Wnt was shown to inhibit Wnt and $\beta$-catenin protein expression, as determined using (A) western blot analysis and (B) statistical analysis of Wnt and $\beta$-catenin protein expression of neurocytes following microRNA-381 overexpression. ${ }^{\# \#} \mathrm{P}<0.01$ vs. negative control group; ${ }^{* *} \mathrm{P}<0.01$ vs. microRNA-381 overexpression group. Negative, negative control group; microRNA-381, microRNA-381 overexpression group; Wnt inhibitor, Wnt-C59 (1 $\mu$ mol/l) group; GAPDH, glyceraldehyde 3-phosphate.

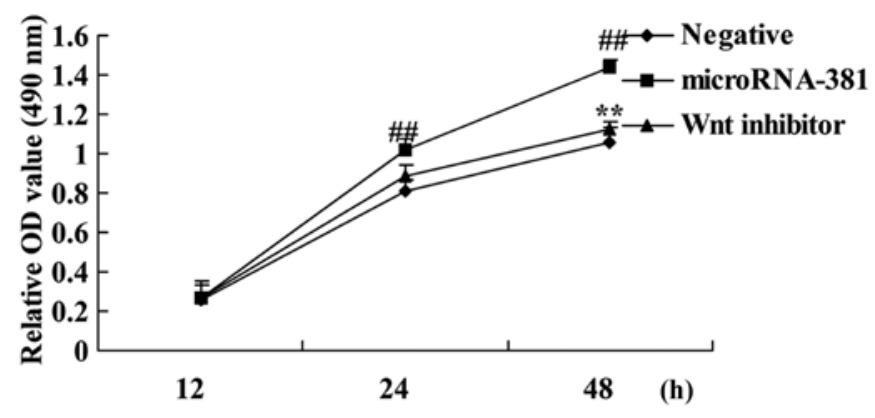

Figure 8. Inhibition of Wnt inhibits cell proliferation of neurocytes following microRNA-381 overexpression. ${ }^{\# \#} \mathrm{P}<0.01$ vs. negative control group; ${ }^{* *} \mathrm{P}<0.01$ vs. microRNA-381 overexpression group. Negative, negative control group; microRNA-381, microRNA-381 overexpression group; Wnt inhibitor, Wnt-C59 (1 $\mu \mathrm{mol} / 1)$ group; OD, optical density.

Overexpression of microRNA-381 suppresses the protein expression of Notch1, NICD and Hesl of neurocytes. To clarify the mechanism underlying the effect of microRNA-381 on Notch1, NICD and Hes1 signaling of neurocytes, Notch1, NICD and Hes1 protein expression was surveyed using western blot analysis. Notch1, NICD and Hes1 protein expression of PC12 cells was significantly suppressed by microRNA-381 overexpression (all $\mathrm{P}<0.05$ ) (Fig. 6).

Inhibition of Wnt inhibits Wnt and $\beta$-catenin protein expression of neurocytes following microRNA-381 overexpression. In order to evaluate the associations between the activation of Wnt signaling and microRNA-381 on SCI, the present study used Wnt inhibitor, Wnt-C59, to suppress Wnt signaling. Wnt inhibitor significantly suppressed the Wnt and $\beta$-catenin protein expression of PC12 cells following overexpression of microRNA-381 compared with the group with microRNA-381 overexpression only (both $\mathrm{P}<0.05$ ) (Fig. 7).

Inhibition of Wnt inhibits cell proliferation of neurocytes following microRNA-381 overexpression. Whether the inhibition of Wnt affects cell proliferation of neurocytes following microRNA-381 overexpression was analyzed. Inhibition of Wnt significantly inhibited the cell proliferation of PC12 cells following microRNA-381 overexpression compared with that of the group with microRNA-381 overexpression only $(\mathrm{P}<0.05)$ (Fig. 8).
Inhibition of Wnt promotes apoptosis and caspase-3 activity of neurocytes following microRNA-381 overexpression. The study now whether the inhibition of Wnt promoted the apoptosis and caspase-3 activity of neurocytes following microRNA-381 overexpression. The apoptosis rate and caspase- 3 activity of PC12 cells with microRNA-381 overexpression were significantly promoted by Wnt inhibitor compared with that in the group with microRNA-381 overexpression only (both $\mathrm{P}<0.05$ ) (Fig. 9).

Inhibition of Wht promotes Hesl and Bax protein expression of neurocytes following microRNA-381 overexpression. To determine whether Wnt participates in the molecular mechanism of microRNA-381 on Hes1 and Bax protein expression of neurocytes, Hes1 and Bax protein expression was measured using western blot analysis. As shown in Fig. 10, the Hes1 and Bax protein expression of PC12 cells upon microRNA-381 overexpression was significantly induced by Wnt inhibitor compared with that of the group with microRNA-381 overexpression only (both $\mathrm{P}<0.05$ ).

Inhibition of Notch suppresses Notch1 and NICD of neurocytes following microRNA-381 overexpression. To further test the associations between the Notch1 signaling pathway and microRNA-381 in SCI, a Notch inhibitor was used to inhibit Notch1 protein expression in the SCI rat model. As shown in Fig. 11, Notch inhibitor, FLI-06, significantly suppressed the Notch1 and NICD protein expression of PC12 cells following microRNA-381 overexpression compared with that of the group with microRNA-381 overexpression only (both $\mathrm{P}<0.05)$.

Inhibition of Notch promotes cell proliferation of neurocytes following microRNA-381 overexpression. Whether the inhibition of Notch promoted the cell proliferation of neurocytes following microRNA-381 overexpression was evaluated. Subsequent to Notch1 signaling pathway inhibition, the cell proliferation of the PC12 cells with microRNA-381 overexpression was significantly promoted compared with that of the group with microRNA-381 overexpression only $(\mathrm{P}<0.05)$ (Fig. 12).

Inhibition of Notch inhibits apoptosis and caspase-3 activity of neurocytes following microRNA-381 overexpression. Whether 
A

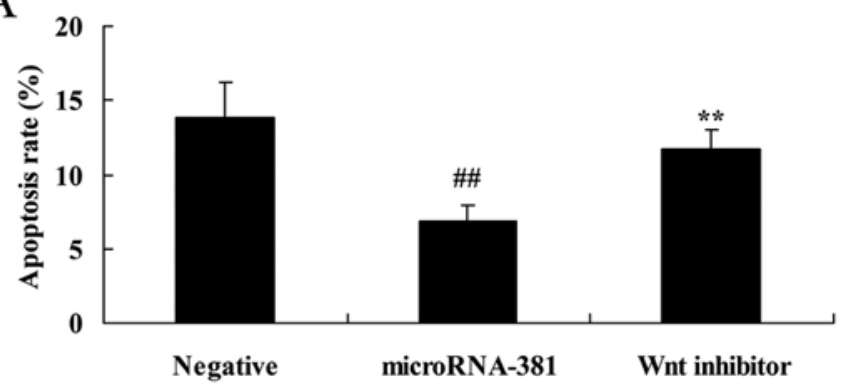

B

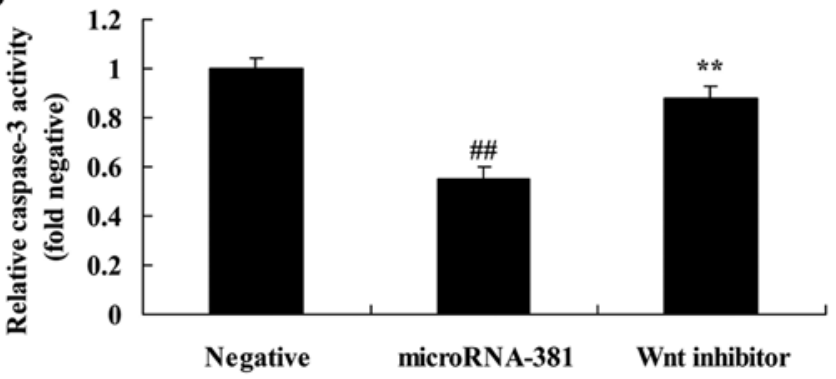

Figure 9. Inhibition of Wnt promotes apoptosis and caspase-3 activity of neurocytes following microRNA-381 overexpression. Inhibition of Wnt was shown to promote

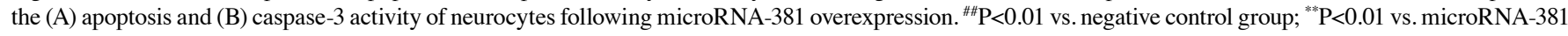
overexpression group. Negative, negative control group; microRNA-381, microRNA-381 overexpression group; Wnt inhibitor Wnt-C59 (1 $\mu$ mol/1) group.

A

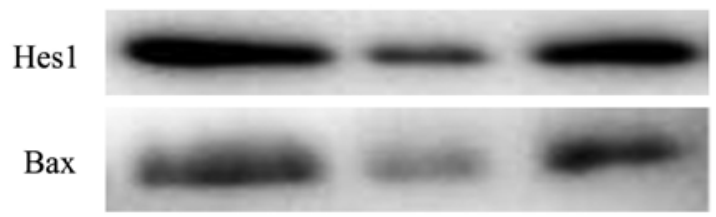

GAPDH

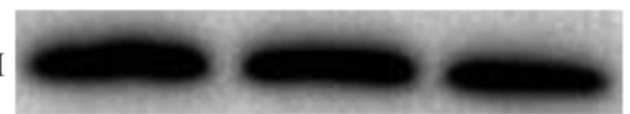

Negative microRNA-381 Wnt inhibitor
$\mathrm{B}$

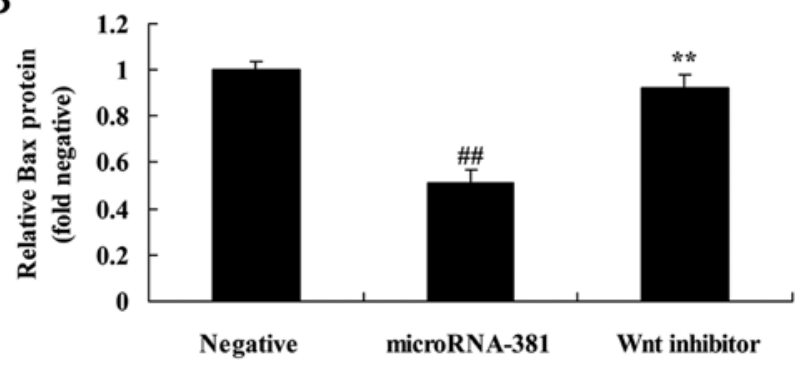

$\mathrm{C}$

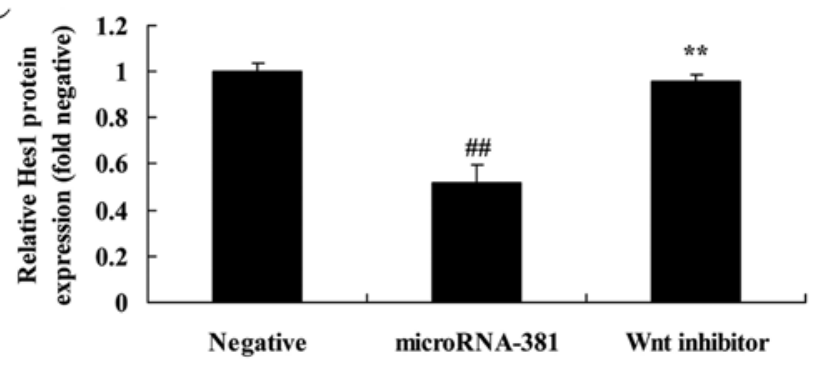

Figure 10. Inhibition of Wnt promotes Hes1 and Bax protein expression of neurocytes following microRNA-381 overexpression. Inhibition of Wnt was shown to inhibit Hes1 and Bax protein expression, as determined using (A) western blot analysis and statistical analysis of (B) Bax and (C) Hes1 protein expression of neurocytes following microRNA-381 overexpression. ${ }^{\# /} \mathrm{P}<0.01$ vs. negative control group; ${ }^{* *} \mathrm{P}<0.01$ vs. microRNA-381 overexpression group. Negative, negative control group; microRNA-381, microRNA-381 overexpression group; Wnt inhibitor Wnt-C59 (1 $\mu$ mol/l) group; GAPDH, glyceraldehyde 3-phosphate.

A

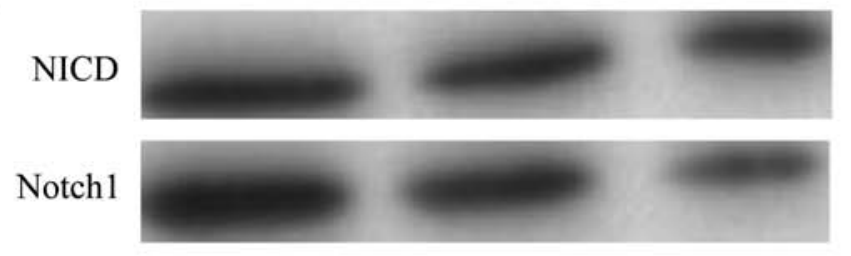

GAPDH

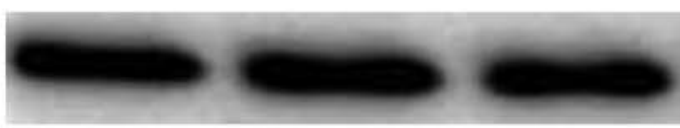

Negative microRNA-381 Notch inhibitor
$\mathrm{B}$

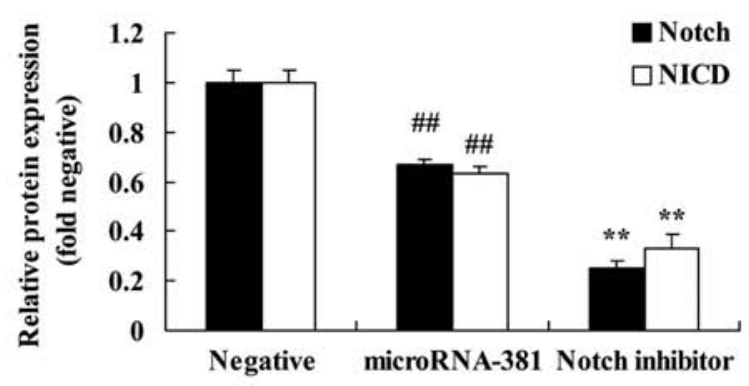

Figure 11. Inhibition of Notch suppresses Notch1 and NICD expression of neurocytes following microRNA-381 overexpression. Inhibition of Notch was shown to suppress Notch1 and NICD protein expression, as determined using (A) western blot analysis and (B) statistical analysis of Notch1 and NICD protein expression of neurocytes following microRNA-381 overexpression. ${ }^{\# \#} \mathrm{P}<0.01$ vs. negative control group; ${ }^{* *} \mathrm{P}<0.01$ vs. microRNA-381 overexpression group. Negative, negative control group; microRNA-381, microRNA-381 overexpression group; Notch1, neurogenic locus notch homolog protein 1; Notch inhibitor, FLI-06 (1 $\mu \mathrm{mol} / 1)$ group; NICD, notch 1 intracellular domain; GAPDH, glyceraldehyde 3-phosphate. 


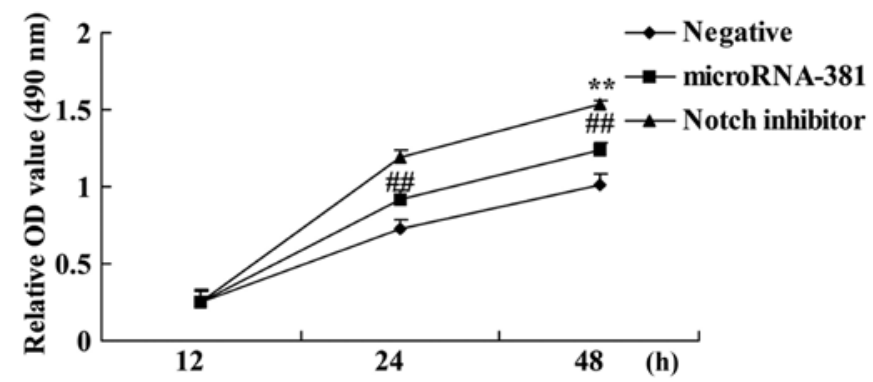

Figure 12. Inhibition of Notch promotes cell proliferation of neurocytes following microRNA-381 overexpression. ${ }^{\# \#} \mathrm{P}<0.01$ vs. negative control group; ${ }^{* *} \mathrm{P}<0.01$ vs. microRNA-381 overexpression group. Negative, negative control group; microRNA-381, microRNA-381 overexpression group; Notch1, neurogenic locus notch homolog protein 1; Notch inhibitor, FLI-06 (1 $\mu \mathrm{mol} / \mathrm{l})$ group; OD, optical density.
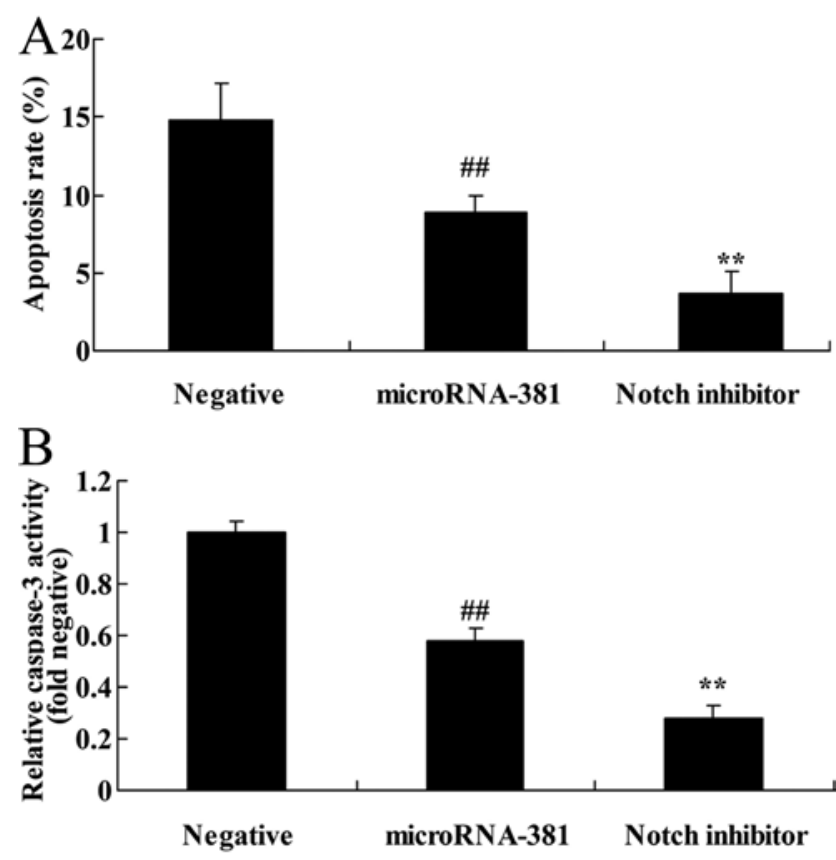

Figure 13. Inhibition of Notch inhibits apoptosis and caspase-3 activity of neurocytes following microRNA-381 overexpression. Inhibition of Notch was shown to inhibite the (A) apoptosis and (B) caspase-3 activity of neurocytes following microRNA-381 overexpression. ${ }^{\# \#} \mathrm{P}<0.01$ vs. negative control group; ${ }^{* *} \mathrm{P}<0.01$ vs. microRNA-381 overexpression group. Negative, negative control group; microRNA-381, microRNA-381 overexpression group; Notch1, neurogenic locus notch homolog protein 1; Notch inhibitor, FLI-06 (1 $\mu \mathrm{mol} / \mathrm{l})$ group.

the inhibition of Notch inhibited the apoptosis and caspase-3 activity of neurocytes following microRNA-381 overexpression was investigated. In PC12 cells following microRNA-381 overexpression, the inhibition of Notch significantly inhibited the apoptosis and caspase- 3 activity of the $\mathrm{PC} 12$ cells compared with that of the group with microRNA-381 overexpression only (both $\mathrm{P}<0.05$ ) (Fig. 13).

Inhibition of Notch inhibits Hes1 and Bax expression of neurocytes following microRNA-381 overexpression. Next, whether Notch inhibition affects the Hes1 and Bax expression of neurocytes following microRNA-381 overexpression was analyzed. Subsequent to microRNA-381 overexpression, Notch inhibition significantly suppressed the Hes1 and Bax protein
A

Bax

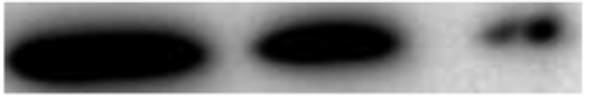

Hes 1

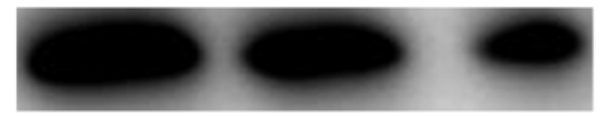

GAPDH

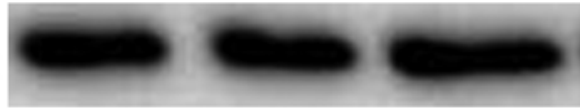

Negative microRNA-381 Notch inhibitor
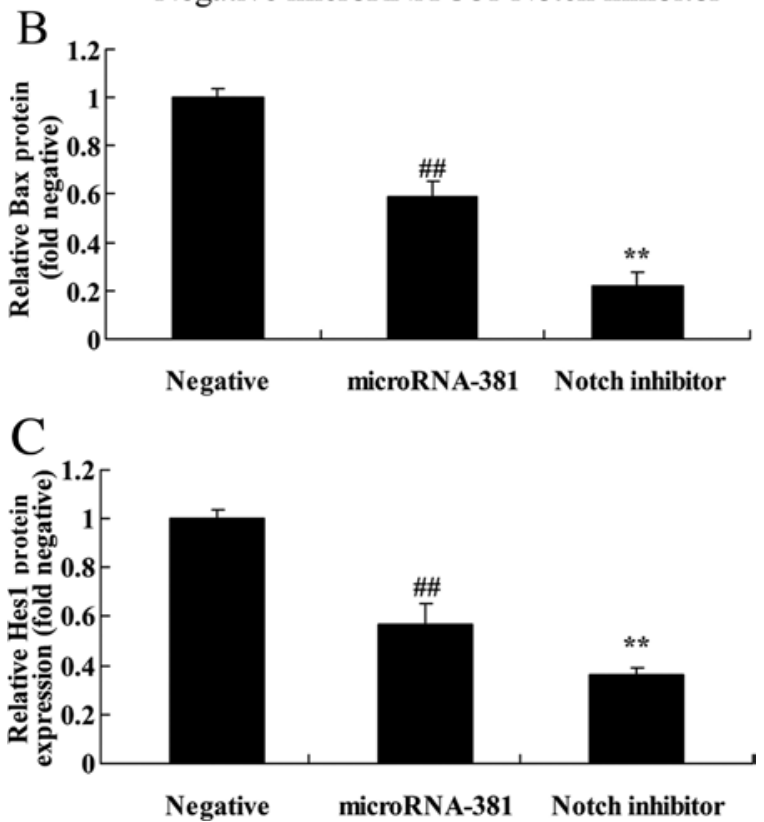

Figure 14. Inhibition of Notch suppresses Hes1 and Bax protein expression of neurocytes following microRNA-381 overexpression. Inhibition of Notch was shown to suppress Bax and Hes1 protein expression, as determined using (A) western blot analysis and statistical analysis of (B) Bax and (C) Hes1 protein expression of neurocytes following microRNA-381 overexpression. ${ }^{\# \#} \mathrm{P}<0.01$ vs. negative control group; ${ }^{* *} \mathrm{P}<0.01$ vs. microRNA-381 overexpression group. Negative, negative control group; microRNA-381, microRNA-381 overexpression group; Notch1, neurogenic locus notch homolog protein 1; Notch inhibitor, FLI-06 (1 $\mu \mathrm{mol} / 1)$ group; Hes1, transcription factor HES-1; Bax, apoptosis and caspase-3 and apoptosis regulator BAX.

expression of the $\mathrm{PC} 12$ cells compared with that of the group with microRNA-381 overexpression only (both $\mathrm{P}<0.05$ ) (Fig. 14).

\section{Discussion}

SCI is the major reason for functional disability, and the resulting huge expenses in aspects such as medical treatment, rehabilitation and nursing, together with the loss of ability to work, have brought heavy burdens on the individual, their family and the country (18). The question of how to take more effective measures to reduce the disability rate of SCI patients, to alleviate the suffering of such patients and to improve their quality of life has become one of the major issues that the orthopedic community faces (19). The present study results suggested that the microRNA-381 expression of model SCI rats was downregulated compared with that of control rats.

SCI is a severe central nervous system trauma (2). MicroRNA (miRNA), which is the micromolecular RNA inhibiting the 


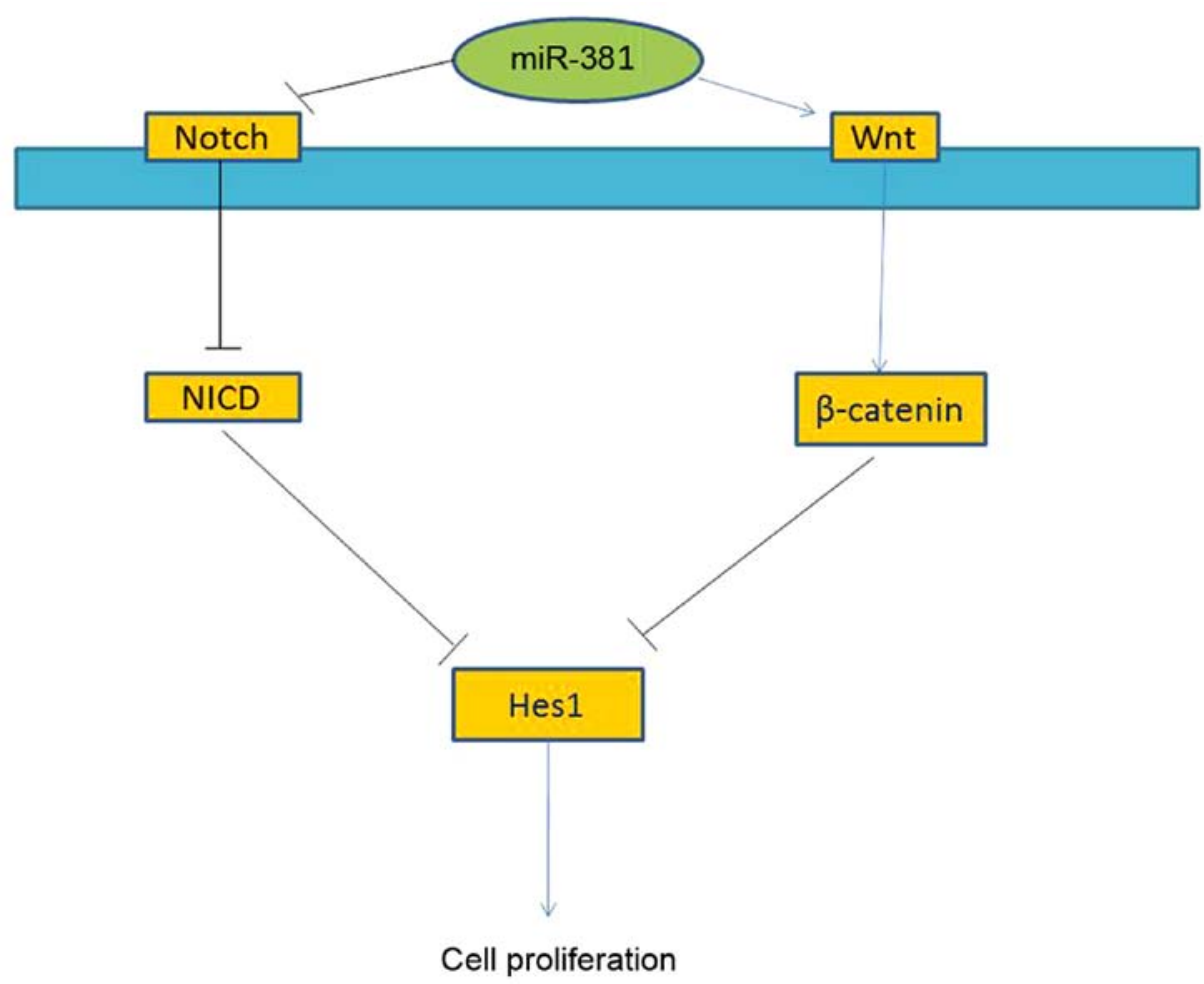

Figure 15. MicroRNA-381 is a potential therapeutic target for SCI acting through Hes1 expression. MicroRNA-381 is a potential therapeutic target for SCI through suppression of Notch1/NICD and induction of Wnt/ $\beta$-catenin by Hes1 expression. SCI, spinal cord injury; Hes1, transcription factor HES-1; Notch1, neurogenic locus notch homolog protein 1; NICD, notch 1 intracellular domain; miR, microRNA.

expression of target genes, serves an important role in regulating relevant genes in processes such as spinal cord development and SCI (2). miRNA may be a novel target for therapeutic intervention that promotes post-SCI nerve repair and regeneration (6). miRNA in SCI remains to be intensively studied, and the mechanisms of action and interaction of miRNAs are important directions of future study (20). This research will aid our understanding of the regulatory role of post-SCI miRNA, and accelerate the development of miRNA regulation-based SCI therapeutic measures (6). In the present study, overexpression of microRNA-381 promoted cell proliferation, and inhibited apoptosis and caspase- 3 and Bax protein expression of neurocytes.

The role of the Notch signal pathway in SCI has attracted increasing attention (21). The Notch signal pathway renders the differentiation of spinal cord-derived stem cells into nerve cells, and inhibits that of neural stem cells into neurogliocytes, which contributes to the therapy for SCI (22). Notch signaling serves an important role in the development of the central nervous system, and Notch is essential for the directional differentiation and maintenance of the neural precursor cells (23). In a recent study, it was found that Notch and its relevant proteins were persistently expressed in the brain hippocampal area of adult mammals (24). Overexpression of Notch can promote the proliferation of neural precursor cells, which has indicated its important role in the regeneration of the central nervous system in adulthood (24). However, the present study only researched the overexpression of microRNA-381 on SCI, thus limitating this study, and further studies will investigate the downregulation of microRNA-381 on SCI in an in vivo or in vitro model.
In recent years, the regulatory effects of Wnt signaling molecule on the proliferation, apoptosis and fate determination of neural tube and neural crest cells in mammalian nervous system have attracted attention (25). It has been found during the embryonic development of Xenopus that inhibiting the Wnt signal pathway can inhibit the formation of the ventral nervous system (25). The deletion of the two signaling proteins Wnt-1 and Wnt-3a will lead to reduced formation of the ventral ganglia (26). Wnt protein could markedly increase the conversion ratio of neural stem cells to neurons (26). Changes in the expression of key factors in the Wnt signal pathway, Wnt-1 and its downstream molecules $\beta$-catenin and glycogen synthase kinase $3 \beta$ (GSK-3 $\beta$ ), following SCI, have been investigated in vivo (27). It can be observed through the expression of these important molecules that the Wnt signal is activated in the early stage of SCI (27). Moreover, the Wnt signaling pathway has been found to serve an important role in the development of the central nervous system, as well as the proliferation and differentiation of neural stem cells, when taking the domestic and overseas research by scholars into consideration (28). The present study results indirectly confirmed that Wnt inhibitor, Wnt-C59 (1 $\mu \mathrm{mol} / \mathrm{l})$, inhibited cell proliferation, promoted apoptosis, and caspase- 3 and Bax protein expression, and suppressed $\beta$-catenin protein expression of $\mathrm{PC} 12$ cells following microRNA-381 overexpression. Zhang et al (29)reported that microRNA-381 suppresses cell growth and invasion through $\mathrm{Wnt} / \beta$-catenin in hepatocellular carcinoma.

It is suggested that the Notch and Wnt signaling pathways participate in the proliferation and differentiation of nerve cells, which even determine cell fates. Notch signal participates in maintaining the undifferentiated state of neuronal 
precursor cells (30). Meanwhile, it regulates the differentiation of neural stem cells into neurons and neuroglia, and may also influence neuronal migration. Hes1 is the most important target gene in the Notch signaling pathway (31). Hes1 protein has been demonstrated to participate in the regulatory process of neural stem cells during the development of the nervous system (31). Hes1-knockout during the neural development of chicken embryos will lead to premature differentiation and maturation of the neural stem cells, resulting in microcephaly or anencephaly (32). Notch cannot inhibit the differentiation of neurons in the absence of Hes1, suggesting that Hes1 is the important effector molecule of the Notch signaling system (32). The present study results indicated that Notch inhibitor, FLI-06 (1 $\mu \mathrm{mol} / \mathrm{l})$, promoted cell proliferation, inhibited apoptosis, and caspase- 3 and Bax protein expression, and suppressed NICD protein expression of PC12 cells following microRNA-381 overexpression. Furthermore, Shi et al (33) showed that microRNA-381 regulates neural stem cell proliferation and differentiation through Hes1.

It has been identified that manual intervention may promote the regeneration of nerve cells, and that moderate trauma can promote the proliferation and differentiation of the neural precursor cells that are in the stationary phase into nerve cells in the hippocampus of the mouse brain (33). In adult mice, the cells that are in the stationary phase (such as somatic stem cells) still have the ability to restart proliferation following years of cell cycle arrest; however, this requires changes in the expression quantity of the Hes1 protein, suggesting that Hes1 protein serves an important role in the development of the somatic cells, particularly the somatic stem cells (23). In addition, microglia activation, astrocyte proliferation and glial scar formation subsequent to SCI are the major factors that hinder the effective regeneration and growth of the injured axons (34). Our previous experimental results also demonstrated that the high expression of Hes1 precedes the maturation of astrocytes during spinal cord development in normal rats, that it serves important roles in the genesis, development and maturation of astrocytes, and that it may promote the differentiation fate of the development of astrocytes (34). In the present study, it was demonstrated that the overexpression of microRNA-381 increased Wnt and $\beta$-catenin protein expression, and suppressed the protein expression of Notch1, NICD and Hes1 of PC12 cells.

The present study demonstrated that overexpression of microRNA-381 promoted the cell proliferation, and inhibited the apoptosis and caspase- 3 and Bax protein expression of neurocytes. Furthermore, its protective effect regulated the Wnt/ $\beta$-catenin and Notch1/NICD signaling pathways by targeting Hes1 in the SCI model (Fig. 15). Collectively, these results provide evidence to suggest that microRNA-381 serves a role in the protective effect against SCI, which could be used in clinical applications.

\section{Acknowledgements}

Not applicable.

\section{Funding}

No funding was received.

\section{Availability of data and materials}

The analyzed data sets generated during the study are available from the corresponding author on reasonable request.

\section{Authors' contributions}

WR designed the experiment. WR, GN, SF, SG and YH performed the experiment. WR analyzed the data. WR wrote the manuscript. All authors read and approved the final manuscript.

\section{Ethics approval and consent to participate}

The Regional Committee for Medical and Health Research Ethics (Health Authority Southern and Eastern Norway) and the Regional Ethics Committee at Tianjin Medical University (Tianjin, China) approved the study protocol.

\section{Consent for publication}

Not applicable.

\section{Competing interests}

The authors declare that they have no competing interests.

\section{References}

1. Wecht JM, Weir JP, Radulovic M and Bauman WA: Effects of midodrine and L-NAME on systemic and cerebral hemodynamics during cognitive activation in spinal cord injury and intact controls. Physiol Rep 4: e1268, 2016.

2. Ziu M, Fletcher L, Savage JG, Jimenez DF, Digicaylioglu M and Bartanusz V: Spatial and temporal expression levels of specific microRNAs in a spinal cord injury mouse model and their relationship to the duration of compression. Spine J 14: 353-360, 2014.

3. Shin JC, Kim KN, Yoo J, Kim IS, Yun S, Lee H, Jung K, Hwang K, Kim M, Lee IS, et al: Clinical trial of human fetal brain-derived neural stem/progenitor cell transplantation in patients with traumatic cervical spinal cord injury. Neural Plast 2015: 630932, 2015.

4. Evans CT, Hill JN, Guihan M, Chin A, Goldstein B, Richardson MS, Anderson V, Risa K, Kellie S and Cameron KA: Implementing a patient education intervention about methicillin-resistant Staphylococcus aureus prevention and effect on knowledge and behavior in veterans with spinal cord injuries and disorders: A pilot randomized controlled trial. J Spinal Cord Med 37: 152-161, 2014.

5. Karthikeyan A, Patnala R, Jadhav SP, Eng-Ang L and Dheen ST: MicroRNAs: Key players in microglia and astrocyte mediated inflammation in CNS pathologies. Curr Med Chem 23: 3528-3546, 2016.

6. Hachisuka S, Kamei N, Ujigo S, Miyaki S, Yasunaga Y and Ochi M: Circulating microRNAs as biomarkers for evaluating the severity of acute spinal cord injury. Spinal Cord 52: 596-600, 2014.

7. Yan $\mathrm{H}$, Hong $\mathrm{P}$, Jiang $\mathrm{M}$ and $\mathrm{Li} \mathrm{H}$ : MicroRNAs as potential therapeutics for treating spinal cord injury. Neural Regen Res 7: 1352-1359, 2012.

8. Strickland ER, Woller SA, Garraway SM, Hook MA, Grau JW and Miranda RC: Regulatory effects of intermittent noxious stimulation on spinal cord injury-sensitive microRNAs and their presumptive targets following spinal cord contusion. Front Neural Circuits 8: 117, 2014.

9. Zhou S, Ding F and Gu X: Non-coding RNAs as emerging regulators of neural injury responses and regeneration. Neurosci Bull 32: 253-264, 2016.

10. Kang SK, Yeo JE, Kang KS and Phinney DG: Cytoplasmic extracts from adipose tissue stromal cells alleviates secondary damage by modulating apoptosis and promotes functional recovery following spinal cord injury. Brain Pathol 17: 263-275, 2007. 
11. Dhanesh SB, Subashini C and James J: Hes1: The maestro in neurogenesis. Cell Mol Life Sci 73: 4019-4042, 2016.

12. Sang L, Roberts JM and Coller HA: Hijacking HES1: How tumors co-opt the anti-differentiation strategies of quiescent cells. Trends Mol Med 16: 17-26, 2010.

13. Aujla PK, Bora A, Monahan P, Sweedler JV and Raetzman LT: The Notch effector gene Hes1 regulates migration of hypothalamic neurons, neuropeptide content and axon targeting to the pituitary. Dev Biol 353: 61-71, 2011.

14. Ingram WJ, McCue KI, Tran TH, Hallahan AR and Wainwright BJ: Sonic Hedgehog regulates Hes1 through a novel mechanism that is independent of canonical Notch pathway signalling. Oncogene 27: 1489-1500, 2008.

15. Baek JH, Hatakeyama J, Sakamoto S, Ohtsuka T and Kageyama R: Persistent and high levels of Hes1 expression regulate boundary formation in the developing central nervous system. Development 133: 2467-2476, 2006.

16. Goto M, Hojo M, Ando M, Kita A, Kitagawa M, Ohtsuka T, Kageyama R and Miyamoto S: Hes1 and Hes5 are required for differentiation of pituicytes and formation of the neurohypophysis in pituitary development. Brain Res 1625: 206-217, 2015.

17. Feng S, Liu W, Zuo S, Xie T, Deng H, Zhang Q and Zhong B: Impaired function of the intestinal barrier in a novel sub-health rat model. Mol Med Rep 13: 3459-3465, 2016.

18. Min K, Oh Y, Lee SH and Ryu JS: Symptom-based treatment of neuropathic pain in spinal cord-injured patients: A randomized crossover clinical trial. Am J Phys Med Rehabil 95: 330-338, 2016

19. Ellis AG, Zeglinski PT, Brown DJ, Frauman AG, Millard M and Furness JB: Pharmacokinetics of the ghrelin agonist capromorelin in a single ascending dose Phase-I safety trial in spinal cord-injured and able-bodied volunteers. Spinal Cord 53: 103-108, 2015.

20. Strickland ER, Woller SA, Hook MA, Grau JW and Miranda RC: The association between spinal cord trauma-sensitive miRNAs and pain sensitivity, and their regulation by morphine. Neurochem Int 77: 40-49, 2014.

21. Guo ZP, Huang MN, Liu AQ, Yuan YJ, Zhao JB and Mei XF: Buyang Huanwu decoction up-regulates Notch1 gene expression in injured spinal cord. Neural Regen Res 10: 1321-1323, 2015.

22. Chen N, Cen JS, Wang J, Qin G, Long L, Wang L, Wei F, Xiang Q, Deng DY and Wan Y: Targeted inhibition of leucine-rich repeat and immunoglobulin domain-containing protein 1 in transplanted neural stem cells promotes neuronal differentiation and functional recovery in rats subjected to spinal cord injury. Crit Care Med 44: e146-e157, 2016.

23. Zhou QZ, Zhang G, Long HB, Lei F, Ye F, Jia XF, Zhou YL Kang JP and Feng DX: Effect of spinal cord extracts after spinal cord injury on proliferation of rat embryonic neural stem cells and Notch signal pathway in vitro. Asian Pac J Trop Med 7: $562-567,2014$
24. Geng X, Sun T, Li JH, Zhao N, Wang Y and Yu HL: Electroacupuncture in the repair of spinal cord injury: Inhibiting the Notch signaling pathway and promoting neural stem cell proliferation. Neural Regen Res 10: 394-403, 2015.

25. Tural Emon S, Uslu S, Ilgaz Aydinlar E, Irban A, Ince U, Orakdogen M and Gulec-Suyen G: Effects of ozone on spinal cord recovery via Wnt/ $\beta$-catenin pathway following spinal cord injury in rats. Turk Neurosurg: May 5, 2016 (Epub ahead of print). doi: 10.5137/1019-5149.JTN.17508-16.1.

26. Yang Z, Wu Y, Zheng L, Zhang C, Yang J, Shi M, Feng D, $\mathrm{Wu} \mathrm{Z}$ and Wang YZ: Conditioned medium of $\mathrm{Wnt} / \beta$-catenin signaling-activated olfactory ensheathing cells promotes synaptogenesis and neurite growth in vitro. Cell Mol Neurobiol 33: 983-990, 2013

27. Briona LK, Poulain FE, Mosimann C and Dorsky RI: $\mathrm{Wnt} / \beta$-catenin signaling is required for radial glial neurogenesis following spinal cord injury. Dev Biol 403: 15-21, 2015.

28. Strand NS, Hoi KK, Phan TM, Ray CA, Berndt JD and Moon RT: Wnt/ $\beta$-catenin signaling promotes regeneration after adult zebrafish spinal cord injury. Biochem Biophys Res Commun 477: 952-956, 2016.

29. Zhang Q, Zhao S, Pang X and Chi B: MicroRNA-381 suppresses cell growth and invasion by targeting the liver receptor homolog-1 in hepatocellular carcinoma. Oncol Rep 35: 1831-1840, 2016.

30. Shi Y, Shu B, Yang R, Xu Y, Xing B, Liu J, Chen L, Qi S, Liu X, Wang $\mathrm{P}$, et al: Wnt and Notch signaling pathway involved in wound healing by targeting c-Myc and Hes1 separately. Stem Cell Res Ther 6: 120, 2015

31. Dakubo GD, Mazerolle CJ and Wallace VA: Expression of Notch and Wnt pathway components and activation of Notch signaling in medulloblastomas from heterozygous patched mice. J Neurooncol 79: 221-227, 2006.

32. Gui X, Meng Z, McConnell YJ, Liu S, Falck VG, Mack LA and Temple WJ: Differing expression profiles of Notch/enterocyte and Wnt/secretory lineage signallings are associated with morphological diversity of appendiceal tumours. J Clin Pathol 70: 40-50, 2017.

33. Shi X, Yan C, Liu B, Yang C, Nie X, Wang X, Zheng J, Wang Y and Zhu Y: miR-381 regulates neural stem cell proliferation and differentiation via regulating Hes1 expression. PLoS One 10: e0138973, 2015.

34. Wang W, Wang P, Li S, Yang J, Liang X, Tang Y, Li Y, Yang R, Wu Y and Shen $\mathrm{H}$ : Methylprednisolone inhibits the proliferation and affects the differentiation of rat spinal cord-derived neural progenitor cells cultured in low oxygen conditions by inhibiting HIF-1 $\alpha$ and Hes1 in vitro. Int J Mol Med 34: 788-795, 2014. 